\title{
Infertilidade na endometriose: etiologia e terapêutica
}

\author{
Thais de Brito Caldeira \\ Isabela Diniz Serra** \\ Luísa de Castro Inácio** \\ Izabela Bartholomeu Noguéres Terra ${ }^{* * *}$
}

\section{ReSUMO}

A endometriose consiste no alojamento de tecidos endometriais em certas regiões que ficam fora do útero. O número de casos dessa patologia é cada vez maior e é capaz de influenciar a fertilidade feminina. Os sinais e sintomas da patologia foram considerados no estudo, além de fatores biopsicossociais que envolvem as mulheres. Esse trabalho foi baseado em uma revisão bibliográfica a partir de uma seleção de artigos que relatam sobre endometriose e sobre a relação dessa enfermidade com infertilidade na população feminina. A partir da revisão de literatura, essa patologia pode causar modificação quimiotática e anatômica no aparelho de reprodução das mulheres, causando assim a infertilidade, dependendo do nível em que se encontra essa doença. Ademais, o tratamento dessa enfermidade, hormonal ou cirúrgico, é capaz de influenciar no processo saúde-doença da mulher, e ainda, possibilitar uma futura gravidez.

\section{Palavras-chave: Endometriose. Infertilidade. Tratamento. Dor.}

\section{INTRODUÇÃO}

O conceito da endometriose, segundo da Sociedade Européia de Reprodução Humana e Embriologia (ESHRE), é "a presença de tecido do endométrio fora do útero, o que induz uma reação inflamatória crônica, esta condição é predominantemente encontrada em mulheres em idade reprodutiva" (CROSSERA et al., 2010).Nessa patologia,o tecido endometrial se aloja, mais comumente, na superfície peritoneal, ovários e septo retovaginal e em menor frequência no Sistema Nervoso Central, pleura e pericárdio (NACUL; SPRITZER, 2010).

A endometriose é uma doença cada vez mais frequente nas mulheres e tem uma importante relação com a fertilidade feminina. No entanto, a forma que a endometriose causa a infertilidade é incerta (CROSERA et al., 2010). A etiopatogeniadessa doença não é bem definida, no entanto as evidências sugerem que a junção de fatores genéticos, hormonais e imunológicos pode favorecer o surgimento da endometriose (KENNEDY et al., 2005).

Essa enfermidade é considerada uma doença da mulher moderna, uma vez que esta tem priorizado a sua formação profissional em detrimento das gestações, causando redução ou adiamento da gravidez, o que aumenta o número de ciclos menstruais (VILA, 2007;
VILASBOAS, 2008). A explicação acerca da relação gestacional com a progressão da doença está no fato de que a progesterona liberada durante a gravidez ou amamentação diminui as chances do desenvolvimento da doença, pois é capaz de neutralizar possíveis focos de endometriose (BELLELIS et al., 2010).

Além do aumento do número de ciclos menstruais, há outros fatores que predispõem o desenvolver dessa doença como deficiência de vitaminas $\mathrm{E}$ na alimentação da mulher e frequente exposição a substâncias tóxicas, dentre elas, desinfetantes (VILA et al., 2010).

Os principais sintomas que acometem as mulheres que possuem endometriose são a dismenorreia, dor pélvica e a dispareunia (BELLELIS et al., 2011).

O reconhecimento da enfermidade pode ser tardio, uma vez que ela se comporta como uma doença silenciosa. Na maioria das vezes, o diagnóstico é realizado quando se faz investigação de infertilidade conjugal, em um grau avançado da doença. Com isso, faz-se fundamental a investigação da presença desse distúrbio na mulher, para que haja prevenção de posteriores complicações para a população feminina (FIGUEIREDO et al., 2016).

\footnotetext{
* Faculdade Dinâmica do Vale do Piranga, Departamento de Medicina - Ponte Nova, MG. E-mail: thaaiscaldeira@hotmail.com

** Faculdade Dinâmica do Vale do Piranga, Departamento de Medicina - Ponte Nova, MG.

*** Orientadora da Numed da secção saúde da mulher.
} 
Para diagnosticar endometriose é essencial a avaliação laparoscópica, porém, há exames de imagem que podem levar ao diagnóstico dessa doença quando na fase avançada (NACUL; SPRITZER, 2010).

A Endometriose apresenta vários estágios que vão de mínima até grave, o que influencia na forma de tratá-la. É uma doença benigna que normalmente ocorre na menacme e pode ser classificado como superficial ou profunda, o que determina os sintomas e o tipo de tratamento (CROSERA et al., 2010).

Muitos são os sentimentos que podem envolver a paciente desde a suspeita até o tratamento da doença. Com isso, faz-se importante que haja apoio emocional, para a mulher acometida por essa doença, tanto no ambiente hospitalar quanto no domiciliar (CARVALHO et al., 2012).

O objetivo desse artigo é descrever, por meio das literaturas existentes, aspectos epidemiológicos, clínicos e terapêuticos da endometriose, a relação dessa doença com a infertilidade feminina e as consequências sociais e psicológicas dessa patologia na vida da mulher. Esse estudo é muito importante, visto que será mais uma fonte de informações e esclarecimentos sobre uma doença que é importante causa da infertilidade feminina.

\section{RevisÃo DE Literatura}

A endometriose é conceituada pela presença de tecido endometrial fora da cavidade uterina, podendo ser encontrado nos ovários, peritônio, região retrocervical, septo retrovaginal, bexiga, reto e outras porções (CROSERA et al., 2010). Ocorre também em regiões extra pélvicas, como a endometriose torácica, encontrada em tecidos parenquimatosos pulmonares ou da pleura (HARUKI et al., 2007). O tratamento pode ser feito por hormônios nos casos de Endometriose mínimas e leves, a partir de anticoncepcionais de via oral, enquanto que o tratamento cirúrgico é destinado a Endometrioses consideradas graves ou moderadas, já que nesse caso tem que haver a remoção do endometrioma e dos tecidos próximos que estão afetados (NAVARRO et al., 2006).

$\mathrm{Na}$ endometriose pélvica pode haver ativação dos macrófagos na cavidade peritoneal, favorecendo o aumento de reativos do oxigênio e nitrogênio, gerando o estresse oxidativo. Havendo peroxidação de lipídeos, de seus produtos de degradação e dos produtos formados pela sua interação com lipoproteínas, além de danificação de células mesoteliais, dessa forma induzindo novos focos endometriais. $\mathrm{O}$ estresse oxidativo participa da etiopatogênese da endometriose e pode cursar comprometendo a fertilidade natural e artificial. Ademais,há uma relação entre o aumento da severidade da doença com aumento de hidroperóxidos e diminuição de vitamina E e glutationa (ANDRADE et al., 2010).

A endometriose classificada como profunda acomete a região retrocervical, vagina, intestino, bexiga, ureteres e, com maior frequência, o septo retovaginal. Os exames para verificar a situação das lesões profundas são limitados, sendo assim é necessária a utilização de outros métodos para o diagnóstico e evolução da doença. Atualmente, são utilizados métodos de imagem para a avaliação, como ultrassonografia transvaginal e transabdominal, ressonância magnética e ultrassonografia transretal/ endoscópico (USTR) (PELOGGIA; PETTA, 2011). A endometriose profunda apresenta uma dor crônica cíclica com relação à menstruação, pode apresentar também dispareunia, disquemia e disúria. Em grande parte dos casos avançados desse tipo de endometriose causa uma disfunção em alguns órgãos, levando a obstrução intestinal e ureteral, hematúria, hemorragia no reto e insuficiência renal (SOMIGLIANA; GARCIA-VELASCO， 2015). $\mathrm{Na}$ endometriose profunda infiltrante, as características da imunidade celular parecem predominar, com a ação das células, enzimas e citocinas que provocam adesão, infiltração e manutenção dos focos do tecido ectópico do endométrio (PODGAEC et al., 2010).

Os primeiros sinais da endometriose começam na adolescência, contudo o diagnóstico normalmente ocorre por volta da terceira década de vida da mulher. Há sintomas que podem se relacionar com essa doença que são mais frequentes, como dismenorreia, alterações intestinais, dor pélvica e dispareunia. Há indicativos de endometriose, durante o exame ginecológico, como dor durante mobilização do útero, retroversão uterina ou aumento do volume ovariano. Verificou-se que $30 \%$ a $40 \%$ das mulheres com endometriose são inférteis, isso se deve à distorção da anatomia, a fatores imunológicos e hormonais. Sendo os dois últimos mais comuns nas mulheres com diversos graus de endometriose. Não se sabe ao certo qual o verdadeiro mecanismo pelo qual a endometriose causa a infertilidade (OLIVEIRA et al. , 2015).

Quando a doença é avançada, a distorção da anatomia pélvica, aderências e oclusão tubária apresenta uma óbvia relação causal com a infertilidade. Já a endometriose leve pode causar disfunção ovulatória, foliculogênese comprometida, implante defeituoso, alterações do endométrio ectópico, ambiente peritoneal imunológico anormal e problemas da fase lútea. Entretanto, a maioria das mulheres possui um nível moderado ou leve da endometriose o que não compromete sua fertilidade (CROSERA et al., 2010) 
Para diagnosticar com melhor exatidão a Endometriose, é preciso uma laparoscopia, que pode dimensionar e analisar a posição correta dos focos de endometriose, oque pode gerar uma melhor confiabilidade sobre a existência da doença. Ultrassonografia transvaginal e Ressonância Nuclear Magnética da Pelve com pesquisa para endometriose pode mostrar locais de doença avançada e infiltrativa. (NACUL; SPRITZER, 2010).

A histerossalpingografia estuda as possíveis alterações anatômicas em pacientes com infertilidade. Muitas vezes os achados são específicos e podem ser interpretados como causa de infertilidade, por exemplo, no diagnóstico de endometriose tubária severa, obstrução bilateral de trompas e outras (PEREZ et al., 2001).

Não há um tratamento definitivo para a endometriose devido à sua incerta etiologia. Mas em casos de mulheres inférteis com endometriose, o tratamento clínico, cirúrgico ou técnicas de reprodução assistida podem ser abordados como uma alternativa para uma possível gravidez. O tratamento clínico utiliza medicamentos os quais reduzem a quantidade de estrogênio e, desse modo, leva a pausa da estimulação do tecido endometrial dependente desse hormônio e, então, impede o desenvolvimento da endometriose. Ainda não existe garantia que esse tipo de tratamento reduz a infertilidade, mas tem uma grande importância no tratamento de dor pélvica (CROSERA et al., 2010).

Uma forma de tratarda dor pélvica épelos inibidores de aromatase, que é destinado para mulheres com dor intensa relacionada com endometriose (ATTAR et al. ,2006; MOUSA et al., 2007). O tratamento é feito com Anastrozol (1 mg) ou Oralletrozole (2,5 mg) uma vez por dia. Estes agentes regulam a formação de locais de estrogênio no interior das lesões endometrióticas e ainda inibe a produção de estrogênio nos ovários (ATTAR et al., 2006; BULUN et al., 1999). As desvantagens são perda óssea com o uso prolongado e desenvolvimento de cisto folicular ovariano. É receitado em combinação com um agonista de GnRH ou um contraceptivo estrogênio-progestina oral, para suprimir o desenvolvimento folicular. Para as mulheres que não podem utilizar os agonistas GnRH ou contraceptivos de estrogênio-progestina oral, é utilizado acetato de noretisterona oral $(5 \mathrm{mg})$ como alternativa e atualmente o Dienogeste $(2 \mathrm{mg})$ (AMSTERDAM et al., 2005).

Mais uma forma de tratamento para a dor pélvica se dá pelos agonistas de gonadotropina de liberação do hormônio $(\mathrm{GnRH})$, já que em uma pesquisa concluiu-se que os agonistas da GnRH se ligam a receptores na hipófise e resultam na diminuição do estrogênio, mas essa diminuição pode causar alguns efeitos adversos, como secura vaginal, diminuição da libido, alterações de humor e diminuição da densidade óssea, que podem ser reduzidos por terapia com acetato de noretindrona oral ou uma combinação de estrogênio e progesterona (PFEIFER et al., 2014).

A cirurgia laparoscópica é uma técnica de benefícios significativos para mulheres inférteis por motivo da endometriose. Nessa cirurgia o objetivo é remover todos os tecidos endometriais ectópicos e aderências, a partir de eletrocauterização ou destruição, através do laser, dos implantes endometriais e adesiólise para melhorar a capacidade de fertilização, em casos de endometriose leve ou moderada. A ressecção laparoscópica ou ablação de endometriose mínima e leve melhora fecundidade em mulheres inférteis (CROSERA et al., 2010).

Um estudo multicêntrico que avaliou 241 mulheres inférteis com endometriose mínima a moderada mostrou taxas de gravidez significativamente mais altas no grupo em que foi realizada laparoscopia com ressecção/ablação dos focos de endometriose. Desta forma, após a cauterização dos focos, este grupo poderá ser submetido ao tratamento da infertilidade (BRASIL, 2010).

O tratamento cirúrgico é proposto às mulheres que já fazem uso de medicamentos e não respondem significativamente ao tratamento, e às pacientes que têm muitos sintomas, que possuem obstrução intestinal ou uretral. Esse tipo de tratamento reduz a dor, fornece diagnóstico histológico, mas possui risco de lesão, possível redução ovariana e formação de aderências. A cirurgia pode ocorrer de três formas: conservadora (controla focos de endometriose), definitiva (histerectomia com ou sem ooforectomia) e radical (remoção das lesões visíveis na cirurgia). Estudos indicam melhor qualidade de vida das pacientes com a abordagem radical (PFEIFER et al. , 2014).

O corte transversal do nervo, incluindo a ablação laparoscópica uterossacral do nervo (LUNA) e neurectomiapré-sacral (PSN), são usados para tratar dismenorreia e dispareunia associados à endometriose. LUNA não é eficaz e PSN não é eficaz ao longo do tempo (DUNSELMAN et al., 2014).

Endometrioma é tratado com o escopo de melhorar a subfertilidade, excluir malignidade e proteger o ovário (DUNSELMEN et al., 2014; CHAPRON et al., 2002; ALBORZI et al., 2006). A ingestão elevada de carne vermelha aumenta o risco de desenvolver a patologia, ao contrário acontecem com as verduras e frutas (PARAZZINI, 2004).

A realização da reprodução assistida para o tratamento de infertilidade causada pela Endometriose

HU Revista, Juiz de Fora, v. 43, n. 2, p. 173-178, abr./jun. 2017 
é bastante eficaz. Esse procedimento realiza a estimulação controlada com Citrato de Clomifeno ou gonadotrifinas associadas à inseminação intrauterina e/ou fertilização in vitro e que melhora a fertilidade em pacientes com endometriose de nível mínimo e leve. Mas, essa conduta poderá ser adotada excluindo o fator idade ( $>35$ anos) e falhas de tentativas prévias, ressaltando-se as taxas esperadas de fecundidade por ciclos $(0,09$ a 0,13) (CROSERA et al., 2010).

A maioria das pacientes ao receberem o diagnóstico de endometriose relata o sentimento de insegurança e ansiedade, mas depois que começam o tratamento o sentimento de bem-estar é predominante, além disso, eles fizeram um estudo em que as participantes relataram sofrer mudanças negativas na qualidade de vida depois que começaram o tratamento, como excesso de peso, diversos efeitos colaterais de medicações e estresse relacionado à antecipação da maternidade. Algumas participantes interromperam o tratamento médico, alegando decepção com o mesmo. Mesmo assim, a maioria das mulheres alegou satisfação ao enfrentar o tratamento. O sentimento positivo era maior do que o impacto ao receber o diagnóstico (VILA et al., 2010).

É importante que o profissional da saúde apoie a reação emocional da paciente ao diagnóstico e ao tratamento e, ainda, incentive que haja apoio do companheiro, da família e dos amigos, já que a ausência desses pode favorecer o aparecimento de transtornos emocionais. Além disso, no caso do enfermeiro que esteja acompanhando uma paciente com endometriose, é válido promover ações que valorizem o diálogo, como rodas de conversas com outras pacientes que estão passando pela mesma situação. Essa troca de experiências é fundamental no processo de tratamento (CARVALHO et al., 2012).

\section{Discussão}

Pode-se observar que a endometriose não é uma doença que se estabelece pela presença do tecido endometrial somente dentro da cavidade uterina ou região pélvica, mas também pode ocorrer em outras localidades como nos tecidos parenquimatosos pulmonares, por exemplo. No que tange ao tratamento, poderá ser desde uso de hormônios até de submissão a processo cirúrgico, o que dependerá do grau da doença, que varia de leve a grave (NAVARRO et al., 2006). Nota-se, de acordo com Oliveira (2005), que há um considerável número de mulheres que possuem relação dessa patologia com a infertilidade, sendo de $30 \%$ a $40 \%$ das mulheres e esse estudo ainda relaciona essa deficiência na fertilidade com a nova anatomia que a mulher passa a ter e por fatores como hormônios e imunologia. No entanto, de acordo com
Crosera et al. (2010), essa relação ainda é incerta. Visto isso, faz-se muito importante que haja estudos sobre esse tema.

Quando a endometriose é muito avançada e provoca alterações anatômicas, a relação com a infertilidade é bem clara. Entretanto, a maioria das mulheres possui grau leve ou moderado permitindoas a maior facilidade em engravidar (CROSERA et al., 2010).

Apesar da etiologia da endometriose ser incerta, o diagnóstico pode ser bem preciso principalmente se associar a videolaparoscopia e/ou ultrassonografia com o diagnóstico cirúrgico. (NACUL; SPRITZER, 2010). Além disso, existe métodos bem específicos de diagnosticar mulheres inférteis com endometriose com a histerossalpingografia. (PEREZ et al., 2001).

A partir da confirmação da doença, diversas opções de tratamentos estão disponíveis. Entretanto, nem sempre a terapêutica da doença irá reverter a infertilidade, como o tratamento com medicamentos que reduzem o estrogênio. Já a cirurgia laparoscópica é uma alternativa que melhora a fecundidade nas mulheres inférteis, mas somente nas que possuem o grau moderado ou leve (CROSERA et al., 2010).

A queixa de dor pélvica relatada pelas portadoras de endometriose pode ser tratada com inibidores de aromatases, mas deve ser associado a agonista de GnRH ou um contraceptivo estrogênio-progestina oral, para impedir a formação de cistos causados pelos inibidores. O aumento do número de ciclos menstruais, devido ao contraceptivo, contribui para a dificuldade dessas mulheres de engravidarem (ATTAR et al., 2006; MOUSA et al., 2007).

O tratamento cirúrgico é orientado às pacientes que já tentaram medicamentos, mas o resultado não foi satisfatório e que apresentam muitos sintomas. Essa terapêutica reduz sintomas causados por essa moléstia. A cirurgia pode ser conservadora, definitiva e radical. (PFEIFER et al., 2014). No estudo mostra que a associação de LUNA e PSN há melhora na dismenorreia e na dispareunia juntamente com a endometriose. Mas não são eficazes em longo prazo (DUNSELMAN et al., 2014).

O tratamento do endometrioma tem por objetivo melhorar a fertilidade, reduzir a malignidade e protege o ovário. A terapêutica é feita por anti-inflamatórios não esteroides e hormônios. A acupuntura auricular é eficaz na dismenorreia em conjunto com endometriose (DUNSELMAN et al., 2014; CHAPRON et al., 2002; ALBORZI et al., 2006).

Para infertilidade o melhor tratamento é a reprodução assistida, o procedimento precisa de uma estimulação controlada com Citrato de clomifeno 
ou gonadotrofina em conjunto com inseminação ou fertilização (CROSERA et al., 2010).

Com o diagnóstico de endometriose, a paciente sente insegura e ansiosa, mas fica mais tranquila após o tratamento. As pacientes relatam diminuição da qualidade de vida mesmo após a terapêutica. O profissional da saúde precisa apoiar emocionalmente a paciente tanto no momento do diagnóstico quanto do tratamento. $\mathrm{E}$ incentivar o apoio de pessoas importantes para a paciente. (CARVALHO; CARVALHO, 2004; CUNHA et al., 2008).

\section{Conclusão}

Mesmo sendo uma patologia benigna, a endometriose pode ser responsável por consequências negativas para a vida da mulher como a infertilidade, transtornos psicológicos, emocionais e sociais. Os estágios avançados da doença geram modificações anatômicas no aparelho reprodutor feminino causando a infertilidade. Os transtornos psicológicos, emocionais e sociais são consequências da dor, que interfere na qualidade de vida e gera um impacto negativo na relação social e conjugal. Essa situação agrava ainda mais quando associada à infertilidade. Não há tratamento definitivo para a doença devido à falta de dados satisfatórios no conhecimento da fisiopatologia e controle da doença, o que estabilizaria a progressão e poderia diminuir os sintomas.

Para as mulheres inférteis, devido a endometriose, existe o tratamento clínico e cirúrgico, associado às alternativas de técnica de reprodução assistida para uma possível gravidez.

Para as mulheres inférteis, existe o tratamento clínico e cirúrgico, associado às alternativas de técnica de reprodução assistida para uma possível gravidez.

\section{Infertility in endometriosis: etiology and treatment}

\section{Abstract}

Endometriosis consists in endometrial tissues growth or adaptation outside of the uterus. The number of cases of this specific pathology has become more frequent and it may be capable influencing female fertility. Biopsychosocial factors as well as signs and symptoms that involve women are considered in this study. This work was based on a bibliographical review of selected articlesthat referred to endometriosis and its relationship with infertility in the female sex. According to this review, this pathology causes chemotactic and anatomic modification in the uterus, and consequently, infertility which depends on how advanced the disease is. Furthermore, the treatment of the disease, hormonal therapy or surgery, can influence the health-disease process as well as enable a future pregnancy.

Key-word: Endometriosis. Infertility. Treatment. Pain.

\section{REFERÊNCIAS}

ALBORZI, S.; ZAREI, A.; ALBORZI, S.; ALBORZI, M. Management of ovarian endometrioma. Clinical Obstetrics \& Gynecology, Shiraz, v. 49, n. 480-491, sep. 2006.

AMSTERDAM, L.L.; GENTRY, W.; JOBANPUTRA, S.; WOLF, M.; RUBIN, S.D.; BULUN, S.E. Anastrozole and oral contraceptives: a novel treatment for endometriosis. Fertility and Sterility, Indianópolis, v. 84, n. 2, p. 300-304, 2005.

ANDRADE, A. Z.; RODRIGUES, J.K.; DIB, L.A.; ROMÃO, G.S.; FERRIANI, R.A.; JUNIOR, A.A.J.; NAVARRO, P.A.A.S. Marcadores séricos de estresse oxidativo em mulheres inférteis com endometriose. Revista Brasileira de Ginecologia e Obstetrícia, vol. 32, n. 6, p. 279-285, jun. 2010.

ATTAR, E; BULUN, S. E. Aromatase inhibitors: the next generation of therapeutics for endometriosis. Fertility and Sterility, Chicago, v. 85, n. 5, p. $1307-1318$, may. 2006.
BELLELIS, P.; PODGAEC, S.; ABRÃO, M.S. Fatores ambientais e endometriose. Revista de Associação Médica Brasileira, São Paulo, v. 57, n. 4, p. 456-461, ago. 2011.

BELLELIS, P.; JUNIOR J.A.D.; PODGAEC, S.; GONZALES, M.; BARACAT, E.C.; ABRÃO, M.S. Aspectos epidemiológicos e clínicos da endometriose pélvica: uma série de casos. Revista de Associação Médica Brasileira, São Paulo, v. 56, n. 4, p. 467-471, may. 2010.

BRASIL, Ministério da Saúde. Protocolo Clínico e Diretrizes Terapêuticas, Endometriose. Portaria SAS/MS no 144 de 31 de março de 2010, p. 253-256. Disponível em < http://www.saude. campinas.sp.gov.br/assist farmaceutica/pcdt/endometriose/ MINUTA-de-Portaria-SAS-PCDT-Endometriose-05-07-2016ATUALIZACAO.pdf >. Acesso em 06 jun. 2006.

HU Revista, Juiz de Fora, v. 43, n. 2, p. 173-178, abr./jun. 2017 
BULUN, S. E., ZEITOUN, K., TAKAYAMA. K; NOBLE, L; MICHAEL, D.; SIMPSON, E.; JOHNS, A.; PUTMAN, M.; SASANO, H. Estrogen production in endometriosis and use of aromatase inhibitors to treat endometriosis. Endocrine-related câncer, Chicago, v. 6, n. 2, p. 293-301, jun. 1999.

CARVALHO, L.F.P.; BELOW, A; ABRÃO, M.S.; Agarwal, A. Minimal and mild endometriosis negatively impact on pregnancy outcome. Revista de Associação Médica Brasileira, São Paulo, v. 58, n. 5, p. 607-614, oct. 2012.

CHAPRON, C.; VERCELLINI, P.; BARAKAT, H.; VIEIRA, M.; DUBUISSON, J.B. Management of ovarian endometriomas. Human Reproduction, Oxford, v. 8, n. 06, p. 591-597, nov. 2002.

CROSERA, A.M.L.V.; VIEIRA, C.H.F.; SAMAMA, M.; MARTINHAGO, S.D.; UENO, J. Tratamento da endometriose associada à infertilidade - revisão da literatura. Femina, São Paulo, v. 38, n. 5, p. 252-256, may. 2010.

DUNSELMAN, G.A., VERMEULEN, N., BECKER, C., CALHAZ, J. C., D'HOOGHE, T.; DE BIE. B.; HEIKINHEIMO O.; HORNE. A.W.; KIESEL, L.; NAP, A.; PRENTICE, A.; SARIDOGAN, E.; SORIANO, D.; NELEN, W. ESHRE guideline: management of women with endometriosis. Human Reproduction, Oxford, v. 29, n. 3, p. 400-412, mar. 2014.

FIGUEIREDO, K.C.F.; COSTA, S.M.C.; RODRIGUES, K.M.; ARRUDA, C.S.L. Endometriose: Uma Doença Silenciosa. In: CONGRESSO BRASILEIRO DE CIÊNCIAS DA SAÚDE, 2016, Campina Grande. Anais eletrônicos I CONBRACIS. Disponível em: <http://editorarealize.com.br/revistas/ conbracis/resumo.php?idtrabalho $=523>$. Acesso em 19 nov. 2016.

HARUKI, T.; FUJIOKA, S.; ADACHI, Y.; MIWA, K.; TANIGUCHI, Y.; NAKAMURA, H. Successful video-assisted thoracic surgery for pulmonary endometriosis: Report of a case. Surgery Today, Tokio, v. 37, n. 2, p. 141-144, jan. 2007.

KENNEDY, S.; BERGQVIST, A.; CHAPRON, C.; D'HOOGHE, T.; DUNSELMAN, G.; GREB, R.; HUMMELSHOJ, L.; PRENTICE, A.; SARIDOGAN, E. Special Interest Group for Endometriosis and Endometrium Guideline Development Group. Guideline for the diagnosis and treatment of endometriosis. Human Reproduction, Oxford, v. 20, n. 10, p. 2698-2704, jun. 2005.

MOUSA, N.A.; BEDAIWY, M.A.; CASPER, R.F. Aromatase inhibitors in the treatment of severe endometriosis. Obstetrics \& Gynecology, Cheveland, v. 109, n. 06, p. 1421-1423, mar. 2007.

NACUL, A.P.; SPRITZER, P.M. Aspectos atuais do diagnóstico e tratamento da endometriose. Revista Brasileira de Ginecologia e Obstetrícia, Rio de Janeiro, v. 32, n. 6, p. 298-307, jun. 2010.

NAVARRO, P.A.A.S.; BARCELOS, I.D.S.; ROSA E SILVA, J.C.Tratamento da endometriose. Revista Brasileira de Ginecologia e Obstetrícia, Rio de Janeiro, v. 28, n. 10, p. 612623, out. 2006.
OLIVEIRA, R.; MUSICH, D.S.; FERREIRA, M.P.S.F.; VILARINO, F.L.; BARBOSA, C.P. Perfil epidemiológico das pacientes inférteis com endometriose. Reprodução e climatério, Santo André, v. 30, n. 1, p. 5-10, mar. 2015.

PARAZZINI, F.; CHIAFFARINO, F.; SURACE, M., CHATENOUD1, L.; CIPRIANIL, S.; CHIANTERAL, V.; BENZI, G.; FEDELE, L. Selected food in take and risk of endometriosis. Human Reproduction, Oxford, v. 19, n. 08, p. 1755-1759, aug. 2004.

PELOGGIA, A.; PETTA, C. A. Endometriose profunda: como abordar? Femina, Campinas, v. 39, n. 9, p. 451-457, set. 2011.

PEREZ, J.A., MAURER, M.N.; ABREU, M.; PITREZ, L.H.; PELLANDA, R.C.; SÈRGIO AUGUSTO CARDOSO MAURER, S.A.M.;FURTADO, A.P.A.; GENRO, C.H. Prevalência de alterações uterinas e tubárias na histerossalpingografia em mulheres inférteis: estudo de 48 casos. Radiologia Brasileira, São Paulo, v. 34, n. 2, p. 79-81, abr. 2001.

PODGAEC, S.; JUNIOR, J. A. D.; CHAPRON, C.; OLIVEIRA, R.M.; BARACAT, E. C.; ABRÃO, M.S. Th1 and Th2 immune responses related to pelvic endometriosis. Revista de Associação Médica Brasileira, São Paulo, v. 56, n. 1, p. 92-98, oct. 2010.

PFEIFER, S.; REINDOLLAR, R.; GOLDBERG, J.; LOBO, R.; THOMAS, M.; PISARSKA, M.; WIDRA, E.; LICHT, M.; SANDLOW, J.; COLLINS, J.; CEDARS, M.; ROSEN, M.; VERNON, M.; DAVIS, O.; DUMESIC, D.; GRACIA, C.; CATHERINO, W.; ODEM, R.; THORNTON, K.; REBAR, R.; LA BARBERA, A. Practice Committee of the American Society for Reproductive Medicine. Treatment of pelvic pain associated with endometriosis: a committee opinion. Fertility and Sterility, Birmingham, v. 101, n. 4, p. 927-935, apr. 2014.

SOMIGLIANA, E.; GARCIA-VELASCO, J.A. Treatment of infertility associated with deep endometriosis: definition of therapeutic balances. Fertility and Sterility, Milan, v. 104, n. 4, p. $764-770$, oct. 2015

VILA, A.C.D. A endometriose e sua relação com a infertilidade feminina e fatores ambientais. 2007. $70 \mathrm{f}$. Dissertação (Mestrado em Ciências Ambientais e Saúde) Universidade Católica de Goiás, 2007.

VILA, A.C.D.; VANDENBERGHE, L.; SILVEIRA, N.A. A vivência de infertilidade e endometriose: pontos de atenção para profissionais de saúde. Psicologia: Saúde \& Doenças, v. 11, n. 2, p. 219-228, nov. 2010.

VILASBOAS, B. Endometriose, doença que causa infertilidade nas mulheres. Webartigos. Disponível em: <http://www. webartigos.comendometriose-doenca-que-causa-infertilidadenas-mulheres $>$. Acesso em 17 abr. 2013.

Enviado em 18/05/2017 Aprovado em 09/08/2017 